5-point scale because the data being analysed were themselves summary statistics. However, due to the discrete nature of the data, and to the question of how different intervals on the fixed point scales related to each other, it would, alternatively, have been appropriate to adopt a non-parametric approach.

Mental Health Clinic

Surfers Paradise, Australia

M. CRAFT

Leicester Frith Hospital

I. A. ISMAIL

Ely Hospital

Brockhall Hospital, Blackburn

Leavesden Hospital

Abbots Langley

Warlingham Park Hospital

Surrey

Applied Statistics Research Unit

Mathematical Institute

University of Kent, Canterbury, Kent

P. M. NORTH

\section{Gilles de la Tourette's Syndrome in Down's Syndrome}

SIR: We recently documented the occurrence and treatment of Gilles de la Tourette's syndrome (GTS) in an individual with Down's syndrome (Journal, May 1986, 148, 601-604). Corbett (Journal, April $1987,150,569)$ suggests that carbamazepine may have accounted for the late onset of GTS in this patient. However, as we indicated in the case report, the patient had been observed by nursing staff to have consistently displayed motor and phonic tics since the age of 11 , when she was first institutionalised. We also mentioned that GTS had been diagnosed according to DSM-III criteria, which stipulate that age of onset must occur between 2 and 15 years. In contrast, carbamazepine had only first been used by this patient when she was aged 24 , following the onset of a possible seizure disorder.

Corbett correctly points out that pharmacological compounds are increasingly being recognised as precipitants of tics. As indicated in the case report, although we attempted to correlate the presence and frequency of tics to the patient's previous use of medication we felt unable to do so accurately in view of the limited information available. However, tics appeared to have been continuously present since at least the age of 11 , an interval that spanned the noncontinuous use of several medications specified in the case report, and antedated the introduction of carbamazepine by 13 years. Although carbamazepine possibly exacerbated her symptomatology, it was clearly not responsible for the onset of GTS manifestations in this patient.

Department of Psychiatry

Toronto General Hospital

Toronto, Ontario

Canada M5G 2 C4

H. KARLINSKY

Surrey Place Centre

Toronto, Ontario

Canada M5S 2C2

J. M. BERG

\section{Onset of Schizophrenia in Men and Women}

SIR: Stromgren has referred to studies indicating that onset of schizophrenia occurs later in women (Journal, January 1987, 150, 1-7). Our experience in India is different.

We have been engaged in a multicentre 5-year follow-up study of schizophrenia, sponsored by the Indian Council of Medical Research, to find out factors associated with the course and outcome of schizophrenia. The centres selected for this study were Lucknow, Madras, and Vellore. All patients who attended psychiatry clinics in the participating centres between 15 October 1981 and 15 October 1982 and who satisfied a modified form of Feighner's criteria for the diagnosis of schizophrenia were included in the study (245 men and 141 women) (Verghese et al, 1985). The age of onset was calculated from the age of the patient at the time of inclusion and the time when the earliest abnormality was noticed (duration of illness). Both these pieces of information were given by the close relatives of the patients. Age of onset was found to be $25.87 \pm 6.67$ years for men and $25.89 \pm 7.33$ years for women, which suggests that age of onset of schizophrenia is not later in women in all parts of the world.

Abraham Verghese JACOB K. JOHN

Christian Medical College

Vellore

S. RAJKUMAR

Madras Medical College

Madras

B. B. SETHI

Sanjay Gandhi Institute of Medical Education and Research

Lucknow

King George's Medical College

J. K. TRIVEDI

Lucknow 